\title{
Automatic Calibration of Commercial Optical See-Through Head-Mounted Displays for Medical Applications
}

\author{
Xue $\mathrm{Hu}^{*}$ \\ Mechatronics in Medicine Laboratory, Imperial College London. \\ Fabio Tatti \\ Mechatronics in Medicine Laboratory, Imperial College London.
}

\author{
Fabrizio Cutolo ${ }^{\dagger}$ \\ Department of Information Engineering, University of Pisa. \\ Ferdinando Rodriguez y Baena ${ }^{\S}$ \\ Mechatronics in Medicine Laboratory, Imperial College London.
}

\begin{abstract}
The simplified, manual calibration of commercial Optical SeeThrough Head-Mounted Displays (OST-HMDs) is neither accurate nor convenient for medical applications. An interaction-free calibration method that can be easily implemented in commercial headsets is thus desired. State-of-the-art automatic calibrations simplify the eye-screen system as a pinhole camera and tedious offline calibrations are required. Furthermore, they have never been tested on original commercial products. We present a gaze-based automatic calibration method that can be easily implemented in commercial headsets without knowing hardware details. The location of the virtual target is revised in world coordinate according to the real-time tracked eye gaze. The algorithm has been tested with the Microsoft HoloLens. Current quantitative and qualitative user studies show that the automatically calibrated display is statistically comparable with the manually calibrated display under both monocular and binocular rendering mode. Since it is cumbersome to ask users to perform manual calibrations every time the HMD is re-positioned, our method provides a comparably accurate but more convenient and practical solution to the HMD calibration.
\end{abstract}

Index Terms: Human-centered computing-Human computer interaction (HCI) - Interaction paradigms - Mixed/augmented reality; Computing methodologies-Computer graphics-Graphics systems and interfaces-Mixed/augmented reality

\section{INTRODUCTION}

Augmented reality (AR) is quickly becoming a powerful tool for Image-Guidance Surgery (IGS). Within this context, a virtual model created from medical scans (e.g., Computer Tomography or Magnetic Resonance Imaging) is superimposed on the surgical site. Surgeons can thus see the patient-specific anatomical model and follow a preoperative plan with better accuracy, reduced invasiveness and a simultaneous view of the real scene. The Optical See-Through head-mounted displays (OST-HMDs) are preferred for IGS as they offer better immersion, safeness and egocentrism. For reliable AR assistance, display calibration, which aligns virtual contents with the perceived reality, is of the utmost importance [3].

The advance in optics design and computational power brings many affordable and highly-compact commercial headsets into the market. Their display calibrations mainly rely on manual virtualto-real alignment by users which are either tedious or inaccurate.

\footnotetext{
*e-mail: xue.hu17@imperial.ac.uk

†e-mail: fabrizio.cutolo@endocas.unipi.it

†e-mail: f.tatti@imperial.ac.uk

§e-mail: f.rodriguez@imperial.ac.uk
}

Furthermore, calibrations are often simplified to improve usability, resulting in suboptimal spatial alignment. While this is tolerable for "gaming" or non specialised experience, calibration must be improved for surgical applications regarding both accuracy and convenience. Automatic calibration is desired in practice. However, state-of-the-art automatic calibration algorithms often require tedious offline calibrations and low-level rendering control, making them hard to be implemented in commercial products [1].

In this paper, we propose an automatic calibration method that can be easily implemented in most commercial OST-HMDs. The modification does not require the access to hardware details of HMDs, and can be done using a universally supported game engine, Unity 3D. Also to the best of our knowledge, this is the first study that demonstrates automatic calibration without the pinhole camera assumption, taking us one step closer to effective automatic calibration of OST-HMDs.

\section{Method}

As shown in Fig.1, if a virtual object is placed at the exact tracked location of a real object $\mathbf{t}$, the rendered pixel $\mathbf{c}^{\prime}$ will not align with $\mathbf{t}$ in user's eye, because of the parallax between the nodal point e and the rendering camera o. Instead of modifying the display within the screen space (i.e. controlling pixel locations in $2 \mathrm{D}$, which may require overriding intrinsic projection matrices), we move the rendered object's location to $\mathbf{t}^{\prime}$, so that the rendered 2D pixel on virtual display $\mathbf{c}$ can lie on the tracked user's gaze $\overrightarrow{e t}$. The modified location can be calculated by $\mathbf{t}^{\prime}=\frac{\overrightarrow{\mathbf{o c}}}{|\overrightarrow{\mathbf{o c}}|} \times|\overrightarrow{\mathbf{t o}}|+\mathbf{o}$.

\section{IMPLEMENTATION}

The Microsoft HoloLens (1st generation) was used for method implementation and performace test. The embedded calibration app requires users to to manually align a finger with six markers displayed on each screen. These alignments are collected to calculate user's interpupillary distance (IPD) which is later utilised for personal parallax correction. Two $640 \times 480$ resolution, $120 \mathrm{fps}$ Pupil Labs eye cameras were rigidly mounted on the HoloLens to track eye location. Unity 3D, a cross-platform game development engine, was used to simplify the AR development.

Fig. 1 shows the overall system configuration. A virtual world coordinate system $W$ is initialised and locked in the physical environment throughout the application's lifetime. A printed ArUco marker serves as the object of interest $\mathbf{t}$. The environment is videoed by the HoloLens front-facing camera $H$. The virtual display is simplified as a 3D plane fixed at a distance $d$ relative to the HMD camera. An error in depth estimation $\Delta d$ will cause a misalignment of $\frac{l}{\frac{d}{\Delta d}+1}$. As $d \gg \Delta d$ and the eye-camera parallax $l$ is usually less than $20 \mathrm{~mm}$ in practice, a $10 \%$ error in depth estimation leads to a hardly noticeable display offset of $1.8 \mathrm{~mm}$ (i.e., around 2 pixels for HoloLens). There- 
fore, we assume $d$ to be HoloLens's reported focal length of $2 \mathrm{~m}$. Required online processing and offline calibrations are summarised as follows:

Online HoloLens location ${ }_{H}^{W}$ T: Provided by HoloLens selftracking capability, accoding to Simultaneous Localization and Mapping (SLAM) algorithm;

Online target location $\mathbf{t}$ : Recorded online video was processed by the OpenCV marker detection algorithm to segment the pixel of interest in real-time. Pixel locations were back-projected into the camera space by intrinsic ${ }_{H} \mathbf{P}$ and further transformed into $W$ by $\mathbf{t}={ }_{H}^{W} \mathbf{T} \times\left({ }_{H} \mathbf{P}\right)^{-1} \times \mathbf{p}_{\mathbf{t}}$

Eye location e: The received real-time eye location in the local tracker coordinate $\mathbf{e}^{(T)}$ was remotely sent to a HoloLens by a python program running on a computer. Data received by the HoloLens were transformed into camera space by the relative camera pose ${ }_{T}^{H} \mathbf{T}$ first, and to the world space subsequently by $\mathbf{e}={ }_{H}^{W} \mathbf{T} \times{ }_{T}^{H} \mathbf{T} \times \mathbf{e}^{(T)}$;

Calibration of HoloLens front-facing camera intrinsic ${ }_{H} \mathbf{P}$ Calibrated with MATLAB (MathWorks inc.) camera calibration toolbox and a printed checkerboard pattern of known size.

Calibration of Eyecam-HoloLens camera pose ${ }_{T}^{H} \mathbf{T}$ : A multimarker system similar to [2] was adopted. The headset was carefuly placed so that both cameras can see at least one marker of the system. The relative camera pose was derived according to the multi-marker relationship and calibrated pose between each marker and camera. 10 trials were carried out. Resultant ${ }_{T}^{H} \mathbf{T}$ shows good consistency, with the maximum translational fluctuation less than 2 $\mathrm{cm}$ and rotational fluctuation less than $6^{\circ}$. The final ${ }_{T}^{H} \mathbf{T}$ is averaged from 10 results.

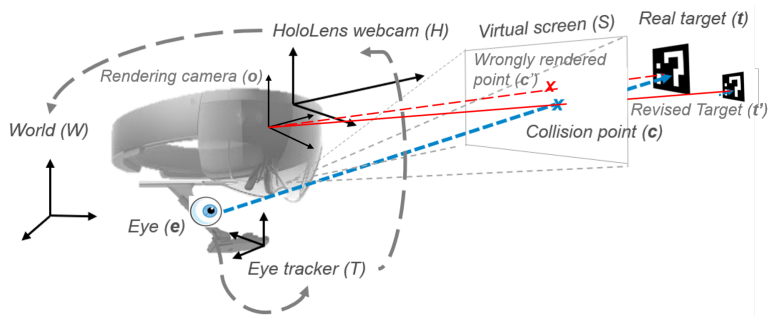

Figure 1: Coordinate transformation between different components within the overall system configuration.

\section{Tests and Results}

Tests under monocular rendering mode was carried out first. The test was repeated twice, with and without a previous standard manual calibration. Users were asked to align a real ArUco marker $\mathbf{t}$ with a virtual cursor $\mathbf{x}$ (with a size of $10 \times 10$ pixel) by moving their head with the support of a chin rest. Meanwhile, according to the tracked location of $\mathbf{t}$, two virtual objects $\mathbf{v}^{\prime}$ and $\mathbf{v}$ were separately updated with and without the proposed display modification. These two objects were hidden from users to avoid distraction. The error is defined as the distance from the rendered pixel of $\mathbf{v}^{\prime}$ or $\mathbf{v}$ to the pixel of $\mathbf{x}$. Cross-subject monocular data were analysed using an ANOVA with post-hoc Tukey's HSD test (Fig. 2). Results show that the accuracy of our modified display is not affected by the pre-calibration status ( $p=0.08$ between "uncali_treat" and "cali_treat" groups), while the display quality of the two untreated groups strongly depends on the pre-calibration status. The automatically calibrated display is much better than the uncalibrated display, demonstrating the effectiveness of our modification. Importantly, the performance of our proposed calibration is statistically equivalent to the standard manual calibration $(p=0.94)$.

For the binocular test, both displays were activated. Users could move their head freely to compare the augmentation quality in terms of visual comfort, spatial alignment and size agreement. Later, they were asked to fill a five-point Likert scale questionnaire that ranged from Strongly Agree to Strongly Disagree. The binocular test was also repeated twice. Subjects' responses were analysed with a twosided Wilcoxon Signed-Rank test. The significance level was set to $5 \%$. With manual calibration, the modified and unmodified displays have similar user-experience, while without previous calibration, the modified display was much better in terms of visual comfort, alignment accuracy and stability.

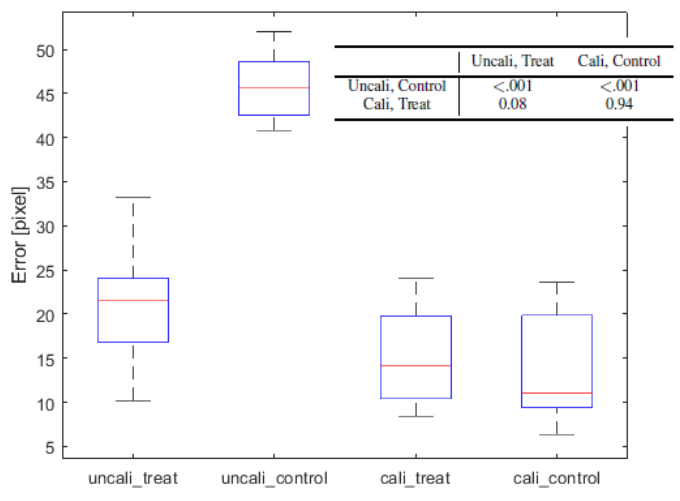

Figure 2: Boxplot of misalignment under 4 conditions with $p$ values.

\section{CONCLUSION}

We propose a method to enable automatic calibration in commercial HMDs via gaze tracking. Experimental trials with the Microsoft HoloLens demonstrate that, when the target is within arm's reach, our modification has comparable accuracy as manual IPD calibration, but is more practical to use. Some other merits include an easy implementation through the display revision in model space, simplified offline calibration, and the potential for automatic display-distortion correction. To further improve the performance of the proposed automatic calibration, offline calibration needs to be improved in the future, and the robust tracking of eye nodal points deserves research. The performance of this method will be compared to the state-of-the-art in the future.

\section{ACKNOWLEDGMENTS}

The authors wish to thank He Liu and Stephen Laws. This work was supported in part by a grant from Imperial College London.

\section{REFERENCES}

[1] J. Grubert, Y. Itoh, K. Moser, and J. E. Swan. A survey of calibration methods for optical see-through head-mounted displays. IEEE Transactions on Visualization and Computer Graphics, 24(9):2649-2662, Sept. 2018. doi: 10.1109/tvcg.2017.2754257

[2] Y. Itoh and G. Klinker. Interaction-free calibration for optical seethrough head-mounted displays based on 3d eye localization. In 2014 IEEE Symposium on 3D User Interfaces (3DUI), pp. 75-82. IEEE, Mar. 2014. doi: 10.1109/3dui.2014.6798846

[3] A. Plopski, Y. Itoh, C. Nitschke, K. Kiyokawa, G. Klinker, and H. Takemura. Corneal-imaging calibration for optical see-through head-mounted displays. IEEE Transactions on Visualization and Computer Graphics, 21(4):481-490, Apr. 2015. doi: 10.1109/tvcg.2015.2391857 